\title{
Safety and tolerability of exenatide once weekly in patients with type 2 diabetes: an integrated analysis of 4,328 patients
}

This article was published in the following Dove Press journal:

Diabetes, Metabolic Syndrome and Obesity: Targets and Therapy 18 May 2015

Number of times this article has been viewed

\author{
Leigh MacConell' \\ Kate Gurney ${ }^{2}$ \\ Jaret Malloy' \\ Ming Zhou ${ }^{3}$ \\ Orville Kolterman ${ }^{4}$ \\ 'Clinical Development, Bristol-Myers \\ Squibb, San Diego, CA, USA; ${ }^{2}$ Medical \\ Writing, Bristol-Myers Squibb, \\ San Diego, CA, USA; ${ }^{3}$ Biostatistics, \\ Bristol-Myers Squibb, Princeton, \\ NJ, ${ }^{4}$ Safety, Bristol-Myers Squibb, \\ San Diego, CA, USA
}

Background: Exenatide once weekly $(\mathrm{QW})$ is a glucagon-like peptide-1 receptor agonist (GLP-1RA) for the treatment of type 2 diabetes. Safety and tolerability are key considerations in treatment selection. This analysis examines the safety and tolerability profile of exenatide QW, other approved GLP-1RAs (exenatide twice daily and liraglutide once daily), and a pooled population of commonly used non-GLP-1RA treatments.

Methods: Intent-to-treat populations from eight randomized Phase III trials with 24-week and 30-week comparator-controlled periods were analyzed. Data were pooled for exenatide QW, exenatide twice daily, and non-GLP-1RA comparator groups; comparisons between exenatide QW and liraglutide were analyzed separately to better match study groups. The incidence of treatment-emergent adverse events with 95\% confidence intervals and exposure-adjusted incidence were calculated. Duration and recurrence were analyzed for gastrointestinal adverse events and adverse events of special interest.

Results: Incidences of serious adverse events did not differ between treatments. Discontinuations due to adverse events occurred numerically less frequently with exenatide QW than with other GLP-1RAs but numerically more frequently than with non-GLP-1RA comparators. The most frequent adverse events in the GLP-1RA groups were gastrointestinal and generally mild, with decreasing incidence over time. Gastrointestinal adverse event incidences appeared lower with exenatide QW versus other GLP-1RAs and greater than with non-GLP-1RA comparators. Injection site-related adverse events seemed highest with exenatide $\mathrm{QW}$, but generally did not lead to withdrawal and abated over time. Hypoglycemia was infrequent overall, but occurred numerically more frequently in the non-GLP-1RA comparator group and increased with concomitant sulfonylurea use. Pancreatitis, thyroid cancer, renal failure, and gallbladder disease were rarely reported.

Conclusion: The overall safety and tolerability profile of exenatide QW was similar to that of other GLP-1RAs, with improved gastrointestinal tolerability. The safety and tolerability profile of exenatide QW compared with non-GLP-1RA comparators was similar overall, with the exception of a lower incidence of hypoglycemia and anticipated differences in gastrointestinal and injection site-related adverse events.

Keywords: glucagon-like peptide-1 receptor agonist, hyperglycemia, adverse events, hypoglycemia

\section{Introduction}

The glucagon-like peptide-1 receptor agonists (GLP-1RAs) are a pharmacologic class of peptide-based, subcutaneously injected, glucose-lowering agents recommended for the treatment of type 2 diabetes as part of combination therapy for glycemic control after initial pharmacologic monotherapy has failed. ${ }^{1-3}$ Key demonstrated benefits of this
Medical Writing, Bristol-Myers Squibb, 9625 Towne Centre Drive,

San Diego, CA 92।21, USA

Tel +l 8585522200

Email kate.gurney@bms.com 
class of agents are reduced glycosylated hemoglobin, reduced postprandial glucose (and fasting glucose depending on duration of action), lowered body weight, and a low incidence of hypoglycemia (without concomitant sulfonylurea use). ${ }^{4-6}$ The unique mechanism of action of GLP-1RAs, ie, targeting the multiple glucose-lowering effects of the hormone glucagonlike peptide-1 (increased glucose-dependent insulin secretion, inhibited glucagon secretion, slowed gastric emptying, and increased satiation), ${ }^{7,8}$ suggests that GLP-1RAs may have some differences in safety and tolerability compared with other diabetes therapies. The safety and tolerability of the GLP-1RAs exenatide twice daily (BID) and liraglutide once daily (QD) have been characterized previously. ${ }^{9-11}$

Exenatide once weekly (QW), the extended-release formulation of exenatide, was approved by the European Medicines Agency in 2011 and by the US Food and Drug Administration (FDA) in 2012 for the treatment of type 2 diabetes. ${ }^{12}$ Exenatide QW is composed of the same parent exenatide molecule as in the BID formulation dispersed in poly-(D,L-lactide-co-glycolide) polymer microspheres. ${ }^{13}$ After subcutaneous injection, the outer polymer shell degrades continuously over an extended period, resulting in an autotitration of exenatide concentration over time. With regular dosing, the minimal effective concentration is exceeded in 2 weeks and steady-state concentration is achieved in 6-7 weeks. ${ }^{7}$

The objective of this integrated retrospective analysis was to evaluate the safety and tolerability profiles of exenatide QW, exenatide BID, liraglutide QD, and the non-GLP-1RA treatments, ie, sitagliptin, pioglitazone, metformin, and insulin glargine, within the exenatide QW drug development program. A pooled database of individual patient data from eight previously reported trials of exenatide QW was used to integrate safety data for 4,328 patients with type 2 diabetes treated for 24 or 30 weeks (blinded-comparator period).

\section{Materials and methods Study participants}

Individual patient data were analyzed from the intent-to-treat populations of eight randomized controlled Phase III trials of exenatide QW, including six trials from the DURATION (DUR; Diabetes Therapy Utilization: Researching Changes in A1C, Weight, and Other Factors Through Intervention With Exenatide Once Weekly) clinical program ${ }^{14-19}$ and two trials conducted solely in Asian populations (Figure 1). ${ }^{20,21}$ Inclusion and exclusion criteria were similar across studies. Concomitant use of other glucose-lowering treatments was limited to stable use within 3 months prior to study screening and was allowed through the duration of the study per protocol. Patients were excluded from study participation if they had used weight loss drugs, other investigational drugs, corticosteroids, drugs known to affect gastrointestinal (GI) motility, transplantation immunosuppression drugs, or confounding treatments for type 2 diabetes within 3 months of screening, or if there was evidence of a clinically significant medical condition.

Patients included in this analysis were treated with exenatide QW $2 \mathrm{mg}$ or an active comparator (exenatide

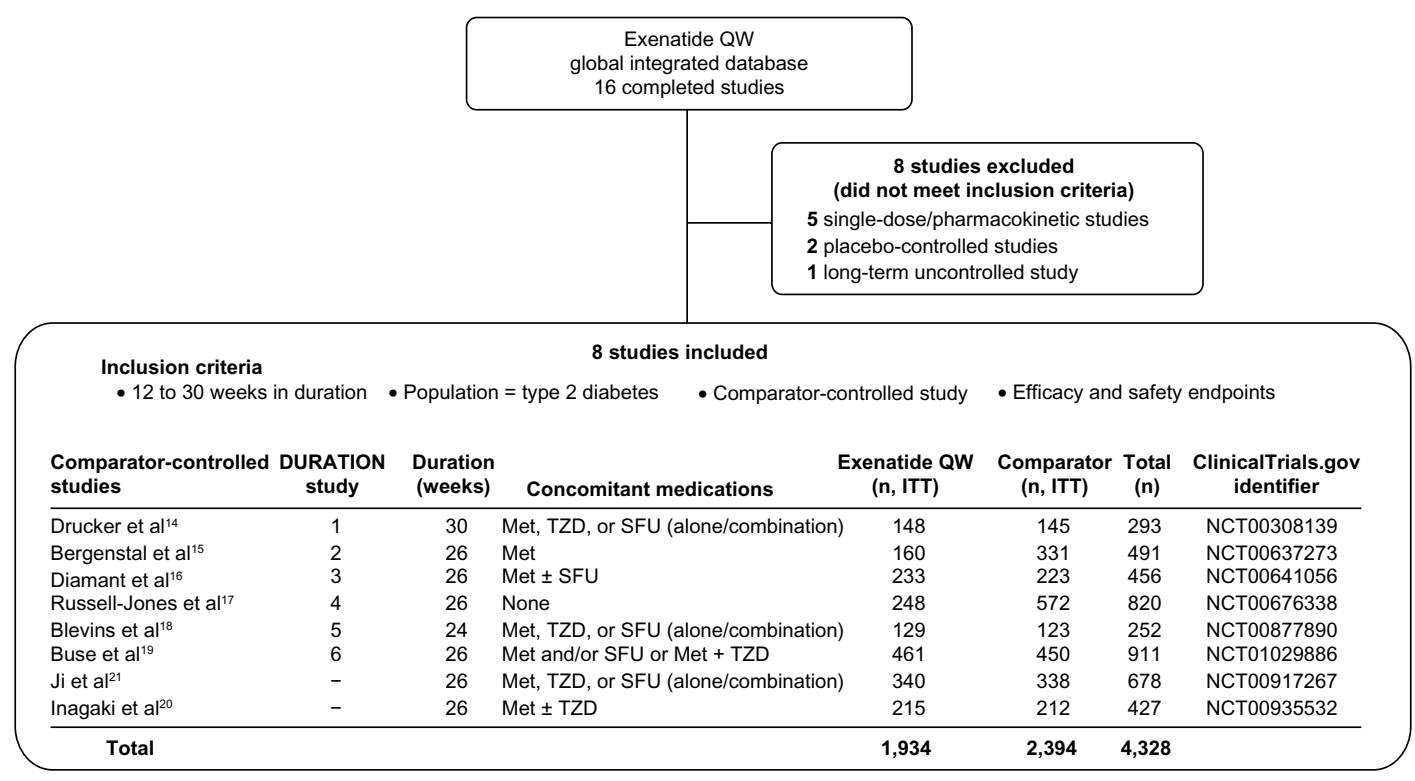

Figure I Selection of trials for pooled analysis and patient disposition. Of the 16 completed clinical trials with available data, eight comparator-controlled studies were included. Abbreviations: ITT, intent-to-treat; Met, metformin; QW, once weekly; SFU, sulfonylurea; TZD, thiazolidinedione; DURATION, Diabetes Therapy Utilization: Researching Changes in AIC, Weight, and Other Factors Through Intervention With Exenatide Once Weekly. 
BID $10 \mu \mathrm{g}$; liraglutide QD $1.8 \mathrm{mg}$; sitagliptin $100 \mathrm{mg}$; pioglitazone $45 \mathrm{mg}$; metformin $2,500 \mathrm{mg}$; or insulin glargine [target fasting blood glucose $72-100 \mathrm{mg} / \mathrm{dL}$ ]). Sitagliptin, pioglitazone, metformin, and insulin were pooled as the nonGLP-1RA comparator group. Details of each of the trials included in this analysis have been reported previously. ${ }^{14-21}$ Concomitant medications used in each study are shown in Figure 1.

Clinical protocols were approved by the institutional review board for each participating study site in accordance with the principles described in the Declaration of Helsinki, including all amendments through the South Africa revision of $1996 .^{22}$ Patients provided written informed consent before study participation.

\section{Safety assessments}

Treatment-emergent adverse events (AEs) were defined as any untoward medical event that either occurred or worsened at any time after the first administration of the study drug through study termination or early termination.

Hypoglycemic episodes were classified as major if they: in the judgment of the investigator or physician, resulted in a loss of consciousness, seizure, or coma and resolved after administration of glucose or glucagon; or required third-party assistance to resolve and had a glucose value of $<54 \mathrm{mg} / \mathrm{dL}$. Minor hypoglycemia was defined as a report of symptoms consistent with hypoglycemia and a glucose value of $<54 \mathrm{mg} / \mathrm{dL}$ prior to treatment of the episode.

Pancreatitis terms included acute and chronic pancreatitis, and the term thyroid neoplasm included benign neoplasm of the thyroid gland and malignant thyroid neoplasm.

Patients who experienced any major adverse cardiac events (MACE) or expanded MACE (defined retrospectively based on individual patient narratives and unadjudicated) during the controlled study periods were assessed. Criteria were based on the Medical Dictionary for Regulatory Activities (MedDRA) version 15.0 standardized MedDRA query terms for MACE, expanded MACE, and cardiac failure. Primary MACE was defined as serious events of cardiovascular death, myocardial infarction (MI), and stroke. Expanded MACE was defined as serious events of cardiovascular death, MI, stroke, unstable angina (classified as serious by the study investigator), heart failure (classified as serious by the study investigator), and transient ischemic attack.

\section{Statistical analysis}

Baseline demographics were summarized for the intent-totreat population (randomized patients who received at least one dose of study treatment) in each treatment group. For the 24-week or 30-week comparator-controlled studies, AEs were reported by preferred term using MedDRA version 14.0 and organized by system organ class. Incidences, exposureadjusted incidence per 100 patient-years, and differences between groups were calculated for comparisons between exenatide QW and liraglutide QD from the DUR-6 study. ${ }^{19}$ Confidence intervals for exposure-adjusted incidence were calculated based on the exact method. Durations of nausea, vomiting, and diarrhea were analyzed by treatment group and over time.

\section{Results \\ Patient characteristics and exposure}

Data from 4,328 patients with type 2 diabetes were pooled from eight clinical studies of exenatide QW (Figure 1), including 1,934 patients treated with exenatide QW, 606 patients treated with exenatide BID, and 1,338 patients treated with a non-GLP-1RA active comparator. The comparison between exenatide QW $(n=461)$ and liraglutide QD $(n=450)$ was analyzed separately and limited to patients who participated in DUR-6 to better match study groups. Table 1 lists the baseline demographic characteristics for the intentto-treat population in each treatment group. Baseline characteristics were generally similar between groups. There were some differences in race between treatment groups due to the inclusion of studies of Asian populations. ${ }^{20,21}$ All groups in the controlled trial period had a similar mean duration of exposure to active treatment of 23-28 weeks.

\section{Patient disposition}

Overall, discontinuations due to AEs were infrequent (Tables 2 and 3, all trials: exenatide QW, 4\%, exenatide BID, $8 \%$, non-GLP-1RA comparator, 2\%; DUR-6: exenatide QW, 3\%, liraglutide QD, 5\%). GI-related AEs were the most common AEs leading to withdrawal in all groups.

\section{Treatment-emergent AEs}

No difference in the incidence of serious AEs or frequency of death was apparent between the treatment groups (Tables 2 and 3).

\section{Gl-related AEs}

GI-related AEs were common in all groups (pooled: exenatide QW, 35\%; exenatide BID, 46\%; non-GLP-1RA comparator, 23\%; DUR-6: exenatide QW, 26\%; DUR-6: liraglutide QD, 42\%). Numerically, nausea and vomiting were reported less frequently with pooled exenatide QW ( $14 \%$ and $7 \%$, respectively) than with exenatide BID (30\% and 13\%; Table 4, Figure 2A and C) but more frequently 
Table I Baseline demographics

\begin{tabular}{|c|c|c|c|c|c|}
\hline \multirow[t]{2}{*}{ Characteristic } & \multicolumn{3}{|c|}{ DURATION-I-6 and Asian studies ${ }^{14-21}$} & \multicolumn{2}{|c|}{ DURATION-6' } \\
\hline & $\begin{array}{l}\text { Pooled exenatide } \\
\text { QW }(n=I, 934)\end{array}$ & $\begin{array}{l}\text { Pooled exenatide } \\
\text { BID }(n=606)\end{array}$ & $\begin{array}{l}\text { Pooled non-GLP-IRA } \\
\text { comparators }(n=I, 338)\end{array}$ & $\begin{array}{l}\text { Exenatide } \\
\text { QW (n=46I) }\end{array}$ & $\begin{array}{l}\text { Liraglutide } \\
\text { QD }(n=450)\end{array}$ \\
\hline Male, \% & 56 & 54 & 58 & 55 & 54 \\
\hline Mean age, years & 55 & 56 & 54 & 56 & 56 \\
\hline \multicolumn{6}{|l|}{ Race/ethnicity, \% } \\
\hline Black/African American & 2 & 5 & 4 & 0.7 & 0.7 \\
\hline White & 48 & 29 & 51 & 66 & 64 \\
\hline Hispanic & 13 & 10 & 12 & 21 & 22 \\
\hline Asian & 37 & 57 & 32 & 12 & 12 \\
\hline Other & 0.2 & 0 & 0.9 & 0.2 & 1 \\
\hline Mean $\mathrm{HbA}_{\mathrm{lc}}, \%(\mathrm{SD})$ & $8.48(1.06)$ & $8.52(1.08)$ & $8.48(1.11)$ & $8.45(1.01)$ & $8.44(1.00)$ \\
\hline Mean BMI, $\mathrm{kg} / \mathrm{m}^{2}(\mathrm{SD})$ & $30.7(5.8)$ & $30.0(5.7)$ & $30.8(5.5)$ & $32.3(5.6)$ & $32.3(5.4)$ \\
\hline $\begin{array}{l}\text { Mean duration of } \\
\text { diabetes, years (SD) }\end{array}$ & $7.1(5.6)$ & $7.8(5.6)$ & $5.3(5.5)$ & $8.0(5.8)$ & $8.8(6.5)$ \\
\hline
\end{tabular}

Abbreviations: BID, twice daily; BMI, body mass index; GLP-IRA, glucagon-like peptide-I receptor agonist; HbA, glycosylated hemoglobin; QD, once daily; QW, once weekly; SD, standard deviation; DURATION, Diabetes Therapy Utilization: Researching Changes in AIC, Weight, and Other Factors Through Intervention With Exenatide Once Weekly.

than in the non-GLP-1RA comparator group (5\% and 3\%). Numerically, diarrhea was reported more frequently with pooled exenatide QW (11\%) than with exenatide BID and the non-GLP-1RA comparator group ( $8 \%$ and $7 \%$, Figure $2 \mathrm{E}$ ). In DUR-6, nausea, vomiting, and diarrhea were reported significantly less often with exenatide QW $(9 \%, 4 \%$, and $6 \%$, respectively) than with liraglutide QD $(21 \%, 11 \%$, and $13 \%$; Table 5, Figure 2A, C and E). The incidence of discontinuations due to GI-related AEs was similar in the pooled exenatide QW and non-GLP-1RA comparator groups (1.4\% and $1.1 \%$, respectively) and numerically higher in the exenatide BID group (5.8\%, Table 2); in DUR-6, rates were $1.3 \%$ and $4.0 \%$ in the exenatide QW and liraglutide QD groups, respectively (Table 3).

The incidence of nausea, vomiting, and diarrhea decreased over time with exenatide QW treatment (Figure 3). Most patients experienced these GI-related AEs within the first weeks of treatment (Figure 3), with few cases recurring.
Although GI-related events typically resolved in fewer than 7 days in most patients across groups, a few patients experienced intermittent events that lasted more than 1 week (Figure 2B, D and F).

\section{Injection site-related AEs}

Injection site-related AEs were reported numerically more frequently with pooled exenatide QW $(20 \%)$ than with exenatide BID $(8.0 \%)$ or the non-GLP-1RA comparator $(8.0 \%)$, and significantly more often with exenatide QW $(16.0 \%)$ than with liraglutide QD (3.0\%; difference, $13.0 \%$; $95 \%$ confidence interval 9.3-16.6) in DUR-6. The incidence of discontinuations due to the combined injection site-related AEs listed above was $0.8 \%$ in the pooled exenatide QW group and $0.0 \%$ in the exenatide BID and non-GLP-1RA comparator groups (Table 2$)$; in DUR-6, there was one patient $(0.2 \%)$ who discontinued exenatide QW due to injection site reactions compared with no discontinuations for liraglutide QD (Table 3).

Table 2 Summary of serious AEs, discontinuations, and deaths for DURATION-I-6 and Asian studies

\begin{tabular}{|c|c|c|c|}
\hline & \multicolumn{3}{|c|}{ DURATION-I-6 and Asian studies ${ }^{|4-2|}$} \\
\hline & $\begin{array}{l}\text { Pooled exenatide } \\
\text { QW }(n=I, 934)\end{array}$ & $\begin{array}{l}\text { Pooled exenatide } \\
\text { BID }(n=606)\end{array}$ & $\begin{array}{l}\text { Pooled non-GLP-IRA } \\
\text { comparators }(n=\mid, 338)\end{array}$ \\
\hline With one or more TEAEs & I,383 (7I.5) (69.50-73.52) & 457 (75.4) (7I.98-78.84) & $880(65.8)(63.23-68.31)$ \\
\hline Serious AEs & $61(3.2)(2.38-3.93)$ & $18(3.0)(1.62-4.32)$ & $60(4.5)(3.38-5.59)$ \\
\hline Deaths & $2(0.1)(0.00-0.25)$ & I (0.2) (0.00-0.49) & $2(0.1)(0.00-0.36)$ \\
\hline Discontinued due to AEs & $85(4.4)(3.48-5.31)$ & $47(7.8)(5.63-9.89)$ & $32(2.4)(1.57-3.2 I)$ \\
\hline Serious AEs & $15(0.8)(0.38-1.17)$ & $3(0.5)(0.00-1.05)$ & $9(0.7)(0.23-1.11)$ \\
\hline Gl-related AEs & $28(1.4)(0.92-1.98)$ & $35(5.8)(3.92-7.63)$ & I5 (I.I) (0.56-I.69) \\
\hline Injection site-related AEs & $16(0.8)(0.42-1.23)$ & $0(0.0)$ & $0(0.0)$ \\
\hline
\end{tabular}

Note: Values are $n(\%)(95 \% \mathrm{Cl})$.

Abbreviations: AEs, adverse events; BID, twice daily; Cl, confidence interval; GI, gastrointestinal; GLP-IRA, glucagon-like peptide-I receptor agonist; QW, once weekly; TEAEs, treatment-emergent adverse events; DURATION, Diabetes Therapy Utilization: Researching Changes in AIC, Weight, and Other Factors Through Intervention With Exenatide Once Weekly. 
Table 3 Summary of serious AEs, discontinuations, and deaths for the DURATION-6 study

\begin{tabular}{|c|c|c|c|}
\hline & \multicolumn{3}{|l|}{ DURATION-6' } \\
\hline & Exenatide QW $(n=46 I)$ & Liraglutide QD $(n=450)$ & Difference $(95 \% \mathrm{Cl})^{\mathrm{a}}$ \\
\hline With one or more TEAEs & $283(61.4)(56.94-65.83)$ & $306(68.0)(63.69-72.31)$ & $-6.6(-12.8,-0.4)$ \\
\hline Serious AEs & $13(2.8)(1.3 \mid-4.33)$ & 7 (I.6) $(0.4 \mathrm{I}-2.70)$ & I.3 $(-0.6,3.2)$ \\
\hline Deaths & $0(0.0)$ & I $(0.2)(0.00-0.66)$ & $-0.2(-0.7,0.2)$ \\
\hline Discontinued due to AEs & $12(2.6)(1.15-4.06)$ & $23(5.1)(3.08-7.15)$ & $-2.5(-5.0,-0.0)$ \\
\hline Serious AEs & $3(0.7)(0.00-1.38)$ & $2(0.4)(0.00-1.06)$ & $0.2(-0.8,1.2)$ \\
\hline Gl-related AEs & $6(1.3)(0.27-2.34)$ & $18(4.0)(2.19-5.81)$ & $-2.7(-4.8,-0.6)$ \\
\hline Injection site-related AEs & I $(0.2)(0.00-0.64)$ & $0(0.0)$ & $0.2(-0.2,0.6)$ \\
\hline
\end{tabular}

Notes: Values are $\mathrm{n}(\%)(95 \% \mathrm{Cl})$ unless otherwise indicated. a Difference = exenatide $\mathrm{QW}$ incidence $(\%)$ minus liraglutide QD incidence (\%).

Abbreviations: AEs, adverse events; $\mathrm{Cl}$, confidence interval; $\mathrm{Gl}$, gastrointestinal; QD, once daily; QW, once weekly; TEAEs, treatment-emergent adverse events; DURATION, Diabetes Therapy Utilization: Researching Changes in AIC, Weight, and Other Factors Through Intervention With Exenatide Once Weekly.

\section{Hypoglycemia}

Hypoglycemia rates were low in all groups except when treatment was combined with a sulfonylurea (Figure 4). Major hypoglycemia occurred in four cases (one each in the pooled exenatide QW and BID groups and two in the pooled comparator group [insulin glargine]). Minor hypoglycemia was reported numerically more often in the non-GLP-1RA comparator group (mainly due to reports in the insulin-treated patients) than in the pooled exenatide QW and exenatide BID groups without concomitant sulfonylurea use ( $4 \%$ versus $2 \%$ and $1 \%$, respectively) and with sulfonylurea use ( $47 \%$ versus $13 \%$ and $12 \%$, respectively). There were no differences in minor hypoglycemia rates for exenatide QW and liraglutide QD in DUR-6.

\section{Other AEs of interest}

Renal failure-related AEs occurred infrequently $(0.1,0.4$, and 0.3 per 100 patient-years for pooled exenatide $\mathrm{QW}$, exenatide
BID, and comparator groups, respectively, and 0.5 and 0.0 per 100 patient-years for exenatide QW and liraglutide QD in DUR-6), with no significant difference between exenatide QW and liraglutide QD (Figure 5). Similarly, thyroid neoplasms were rarely reported in any treatment group $(0.2,0.4$, and 0.5 per 100 patient-years, respectively, and 0.5 and 0.0 for exenatide QW and liraglutide QD in DUR-6; Figure 5). No cases of thyroid cancer or $\mathrm{C}$-cell carcinoma were reported. There were no cases of pancreatic cancer reported in any group analyzed, although one case of pancreatic neoplasm was reported for exenatide BID. Pancreatitis was rare in all groups (Figure 5).

Incidences of serious AEs grouped by body system are shown in Table 6. Serious AEs reported in two or more patients per group for pooled exenatide QW were acute pancreatitis, acute cholecystitis, cholelithiasis, appendicitis, viral pericarditis, cerebral artery occlusion, cerebral infarction, and ureteric calculus (each 2/1,934 [0.1\%]),

Table 4 Summary of frequent ( $\geq 5 \%$ ) treatment-emergent adverse events for DURATION-I -6 and Asian studies

\begin{tabular}{|c|c|c|c|}
\hline \multirow[t]{2}{*}{ Preferred term } & \multicolumn{3}{|c|}{ DURATION-I-6 and Asian studies ${ }^{\mid 4-21}$} \\
\hline & $\begin{array}{l}\text { Pooled exenatide } \\
\text { QW }(n=1,934)\end{array}$ & $\begin{array}{l}\text { Pooled exenatide } \\
\text { BID }(n=606)\end{array}$ & $\begin{array}{l}\text { Pooled non-GLP-IRA } \\
\text { comparators }(n=1,338)\end{array}$ \\
\hline Nausea & 279 (I4.4) (I2.86-15.99) & I8I (29.9) (26.22-33.5I) & 66 (4.9) (3.77-6.09) \\
\hline Diarrhea & $203(10.5)(9.13-11.86)$ & $5 I(8.4)(6.21-10.63)$ & $99(7.4)(6.00-8.80)$ \\
\hline Nasopharyngitis & $183(9.5)(8.16-10.77)$ & $24(4.0)(2.4 I-5.5 I)$ & $152(11.4)(9.66-13.06)$ \\
\hline Vomiting & $130(6.7)(5.6 \mid-7.84)$ & $79(13.0)(10.36-15.72)$ & $33(2.5)(1.64-3.30)$ \\
\hline Injection site induration & $126(6.5)(5.42-7.61)$ & $2(0.3)(0.00-0.79)$ & $12(0.9)(0.39-1.40)$ \\
\hline Constipation & $124(6.4)(5.32-7.50)$ & $38(6.3)(4.34-8.20)$ & $33(2.5)(1.64-3.30)$ \\
\hline Headache & $123(6.4)(5.27-7.45)$ & $28(4.6)(2.95-6.29)$ & $116(8.7)(7.16-10.18)$ \\
\hline Injection site nodule & $119(6.2)(5.08-7.22)$ & $0(0.0)$ & $48(3.6)(2.59-4.58)$ \\
\hline Injection site pruritus & $106(5.5)(4.47-6.50)$ & $12(2.0)(0.87-3.09)$ & 19 (1.4) (0.79-2.05) \\
\hline Decreased appetite & $75(3.9)(3.02-4.74)$ & $38(6.3)(4.34-8.20)$ & $7(0.5)(0.14-0.91)$ \\
\hline Upper respiratory tract infection & $73(3.8)(2.93-4.62)$ & 44 (7.3) (5.19-9.33) & $52(3.9)(2.85-4.92)$ \\
\hline Dyspepsia & $65(3.4)(2.56-4.16)$ & $13(2.1)(0.99-3.30)$ & $33(2.5)(1.64-3.30)$ \\
\hline Dyslipidemia & $59(3.1)(2.28-3.82)$ & $34(5.6)(3.78-7.44)$ & $31(2.3)(1.5 I-3.12)$ \\
\hline Dizziness & $52(2.7)(1.97-3.4 I)$ & $30(5.0)(3.22-6.68)$ & $42(3.1)(2.20-4.07)$ \\
\hline
\end{tabular}

Note: Values are $\mathrm{n}(\%)(95 \% \mathrm{Cl})$.

Abbreviations: BID, twice daily; Cl, confidence interval; GLP-IRA, glucagon-like peptide-I receptor agonist; QW, once weekly; DURATION, Diabetes Therapy Utilization: Researching Changes in AIC, Weight, and Other Factors Through Intervention With Exenatide Once Weekly. 


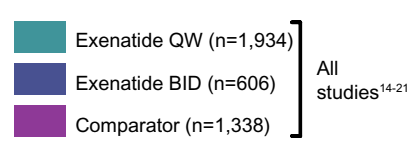

$\left.\begin{array}{l}\text { Lexenatide QW }(n=461) \\ \text { Liraglutide QD }(n=450)\end{array}\right]$ DURATION-6 $6^{19}$

A

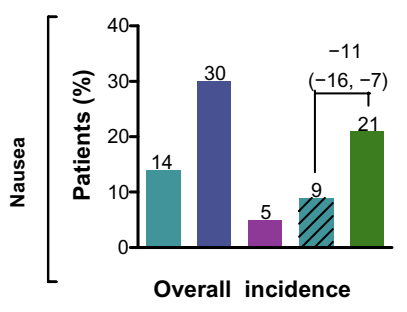

C

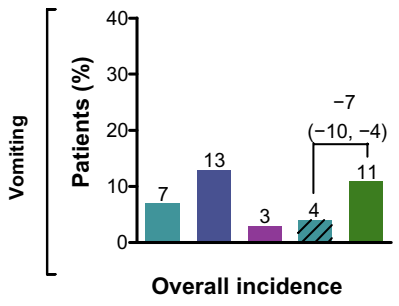

E

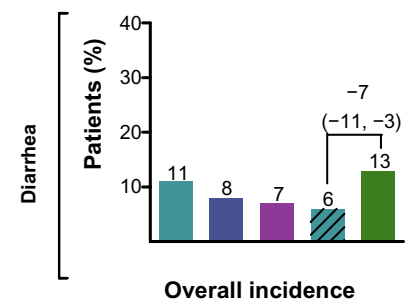

B

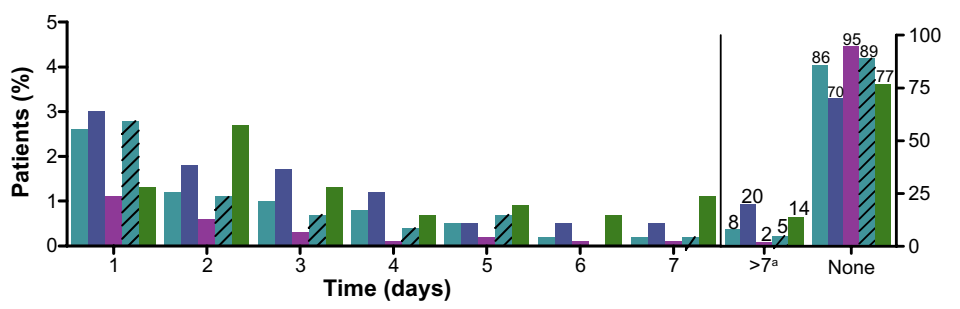

D

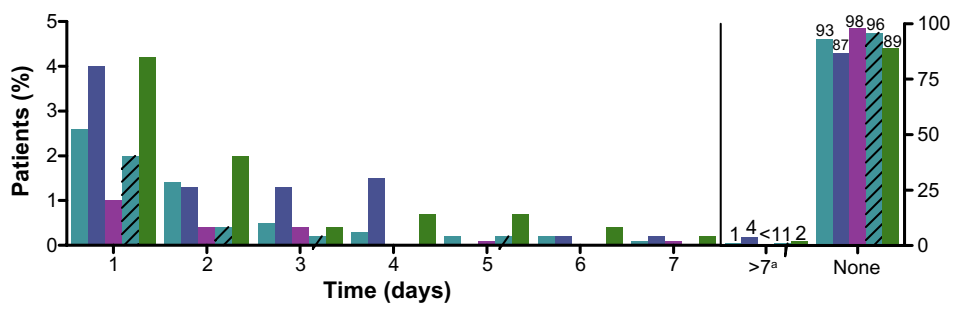

F

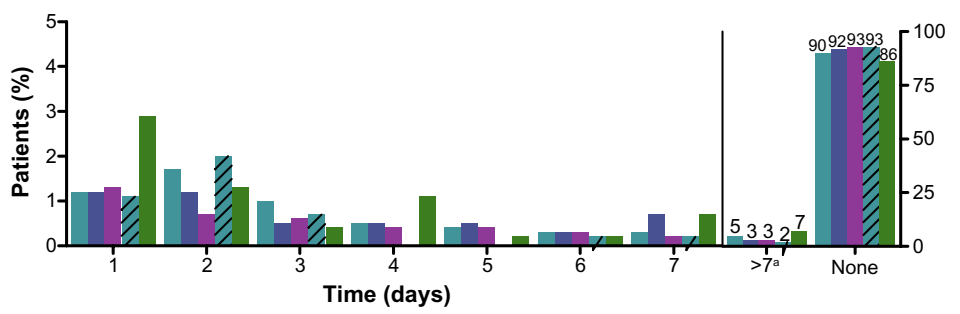

Figure 2 Incidence and duration of gastrointestinal-related adverse events over time. Incidence (left panel) and duration (right panel) for (A, B) nausea, (C, D) vomiting, and (E, F) diarrhea in each treatment group. Duration of the nausea/vomiting event is calculated as the resolution date (or the last participation date if event is ongoing at the time of study termination) minus the event onset date plus I. ${ }^{a}$ Events lasting longer than 7 days in duration included reports of both continuous and intermittent nausea/ vomiting. 95\% confidence interval for the difference (exenatide QW incidence [\%] minus liraglutide QD incidence [\%] in DURATION-6).

Abbreviations: BID, twice daily; QD, once daily; QW, once weekly; DURATION, Diabetes Therapy Utilization: Researching Changes in AIC, Weight, and Other Factors Through Intervention With Exenatide Once Weekly.

Table 5 Summary of frequent $(\geq 5 \%)$ treatment-emergent adverse events for the DURATION-6 study

\begin{tabular}{|c|c|c|c|}
\hline \multirow[t]{2}{*}{ Preferred term } & \multicolumn{3}{|l|}{ DURATION-6 } \\
\hline & Exenatide QW ( $(n=46 I)$ & Liraglutide QD $(n=450)$ & Difference $(95 \% \mathrm{Cl})^{\mathrm{a}}$ \\
\hline Nausea & $43(9.3)(6.67-11.98)$ & 93 (20.7) (I6.93-24.4I) & $-11.3(-15.9,-6.8)$ \\
\hline Diarrhea & $28(6.1)(3.89-8.25)$ & $59(13.1)(9.99-16.23)$ & $-7.0(-10.8,-3.2)$ \\
\hline Nasopharyngitis & 31 (6.7) (4.44-9.0I) & $32(7.1)(4.74-9.49)$ & $-0.4(-3.7,2.9)$ \\
\hline Vomiting & I7 (3.7) (I.97-5.4I) & $48(10.7)(7.8 I-13.52)$ & $-7.0(-10.3,-3.6)$ \\
\hline Injection site induration & NR & NR & NR \\
\hline Constipation & $21(4.6)(2.65-6.46)$ & $22(4.9)(2.90-6.88)$ & $-0.3(-3 . I, 2.4)$ \\
\hline Headache & $27(5.9)(3.7 I-8.00)$ & $38(8.4)(5.88-11.01)$ & $-2.6(-5.9,0.8)$ \\
\hline Injection site nodule & $48(10.4)(7.62-13.20)$ & $5(1 . I)(0.14-2.08)$ & $9.3(6.3,12.3)$ \\
\hline Injection site pruritus & I5 (3.3) (I.63-4.87) & I (0.2) (0.00-0.66) & $3.0(1.4,4.7)$ \\
\hline Decreased appetite & I7 (3.7) (I.97-5.4I) & $29(6.4)(4.18-8.71)$ & $-2.8(-5.6,0.1)$ \\
\hline Upper respiratory tract infection & $12(2.6)(1.15-4.06)$ & $12(2.7)(1.18-4.16)$ & $-0.1(-2.1,2.0)$ \\
\hline Dyspepsia & II (2.4) (0.99-3.78) & $27(6.0)(3.8 I-8.19)$ & $-3.6(-6.2,-1.0)$ \\
\hline Dyslipidemia & NR & NR & NR \\
\hline Dizziness & I3 (2.8) (I.3 I-4.33) & I5 (3.3) (I.67-4.99) & $-0.5(-2.8,1.7)$ \\
\hline
\end{tabular}

Notes: Values are $\mathrm{n}(\%)(95 \% \mathrm{Cl})$ unless otherwise indicated. aDifference = exenatide QW incidence (\%) minus liraglutide QD incidence (\%) in DURATION-6. From Lancet, Volume $38 \mathrm{I}(986 \mathrm{I})$, Buse JB, Nauck M, Forst T, et al, Exenatide once weekly versus liraglutide once daily in patients with type 2 diabetes (DURATION-6): a randomised, open-label study, II7-124, Copyright 2013, with permission from Elsevier. ${ }^{19}$

Abbreviations: $\mathrm{Cl}$, confidence interval; NR, not reported using this term; QD, once daily; QW, once weekly; DURATION, Diabetes Therapy Utilization: Researching Changes in AIC, Weight, and Other Factors Through Intervention With Exenatide Once Weekly. 


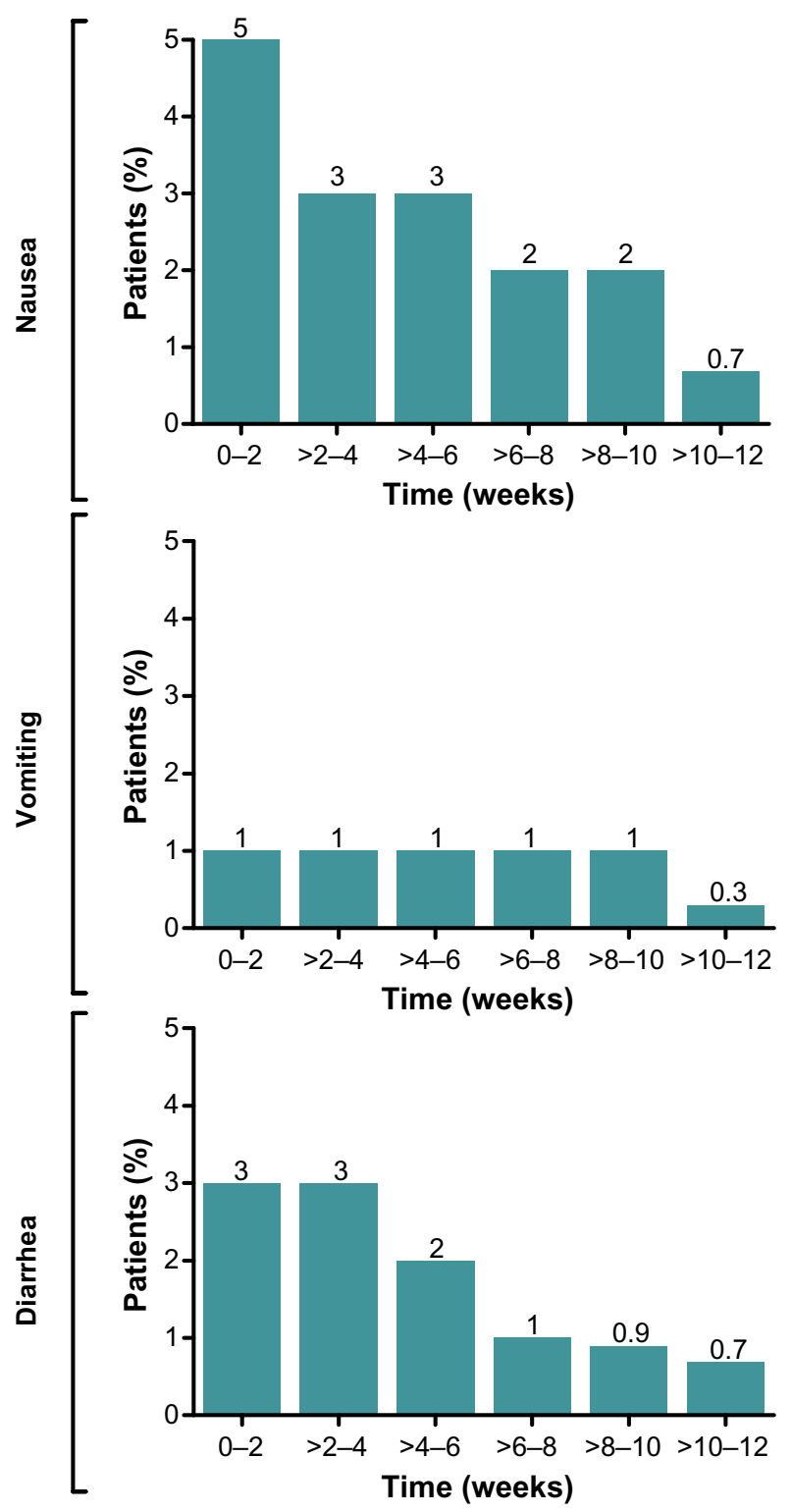

Figure 3 Occurrence of gastrointestinal-related adverse events over time with exenatide QW treatment. Incidences of nausea, vomiting, and diarrhea in patients treated with exenatide QW are combined for weeks 0-12 in 2-week increments. ${ }^{14-21}$ Abbreviation: QW, once weekly.

and prostate cancer $(3 / 1,934[0.2 \%])$. For non-GLP-1RA comparators, serious AEs were angina pectoris, unstable angina, coronary artery occlusion, non-cardiac chest pain, cholecystitis, and cholelithiasis (each 2/1,338 [0.1\%]), and chest pain and cerebrovascular accident (3/1,338 [0.2\%]). Only appendicitis was reported for two or more patients with exenatide QW in DUR-6 (2/461 [0.4\%]). Notably, serious cardiac and vascular disorders were rarely reported (cardiac disorders: $0.4 \%, 0.7 \%, 0.7 \%, 0.2 \%$, and $0.4 \%$, respectively, for pooled exenatide QW, exenatide BID, non-GLP-1RA comparators, DUR-6 exenatide QW, and liraglutide QD; vascular disorders: $0.1 \%, 0.2 \%, 0.1 \%, 0.0 \%$, and $0.0 \%$, respectively).

\section{Cardiovascular risk}

The incidence of primary MACE for all GLP-1RAs and nonGLP-1RAs was $0.6 \%(n=18 / 2,990)$ and $0.5 \%(n=7 / 1,338)$, respectively. Expanded MACE was $0.8 \%(n=25 / 2,990)$ for all GLP-1RAs and $1.3 \%(\mathrm{n}=17 / 1,338)$ for all non-GLP-1RAs. The incidence of primary MACE or expanded MACE by treatment shows a numerically lower incidence with exenatide QW (0.6\% and $0.7 \%$, respectively) and exenatide BID ( $0.5 \%$ and $0.8 \%)$ than with liraglutide QD (0.9\% and $1.3 \%)$. For non-GLP-1 comparators, the numerical incidences of primary MACE and expanded MACE were highest for pioglitazone ( $1.2 \%$ and $2.4 \%$, respectively) followed by sitagliptin $(0.6 \%$ and $0.9 \%)$, metformin $(0.4 \%$ and $1.6 \%)$, and insulin $(0.0 \%$ and $0.5 \%)$.

\section{Discussion}

This analysis of the exenatide QW clinical trial population compared the safety and tolerability profiles of exenatide QW with those of GLP-1RA comparators and commonly used non-GLP-1RA treatments. Consistent with previous reports, no new specific safety signals were identified in this retrospective pooled analysis of the DURATION clinical trials conducted to evaluate the safety and efficacy of exenatide QW. The most common AEs with exenatide QW were GI-related and injection site-related, and were generally mild and transient.

The prevalence of GI-related AEs is a consistent class effect observed with GLP-1RAs, and it is known that GI symptoms are generally more frequent in patients with diabetes compared with those without diabetes. ${ }^{4,23-27}$ The issue of GI tolerability is a key concern with glucoselowering treatments and has the potential to affect treatment adherence and compliance. ${ }^{28}$ Incidences of GI-related AEs were significantly lower with exenatide QW compared with liraglutide QD. The incidence of nausea and vomiting was approximately doubled with liraglutide QD versus exenatide QW. Although GI events with exenatide QW and BID were numerically more frequent than with the non-GLP-1RA comparator group, GI events were most frequent early in treatment and for a short duration before decreasing over time. Importantly, there was no increase in prolonged duration of GI events with long-acting exenatide QW compared with shorter-acting treatments, with the majority of events with exenatide QW having resolved within 7 days. In addition, exenatide QW was associated with a low discontinuation rate due to GI events. As in the present analysis, long-term studies of exenatide BID and exenatide QW have shown that nausea generally subsided with continued treatment. ${ }^{29-31}$ Similarly, other studies of 


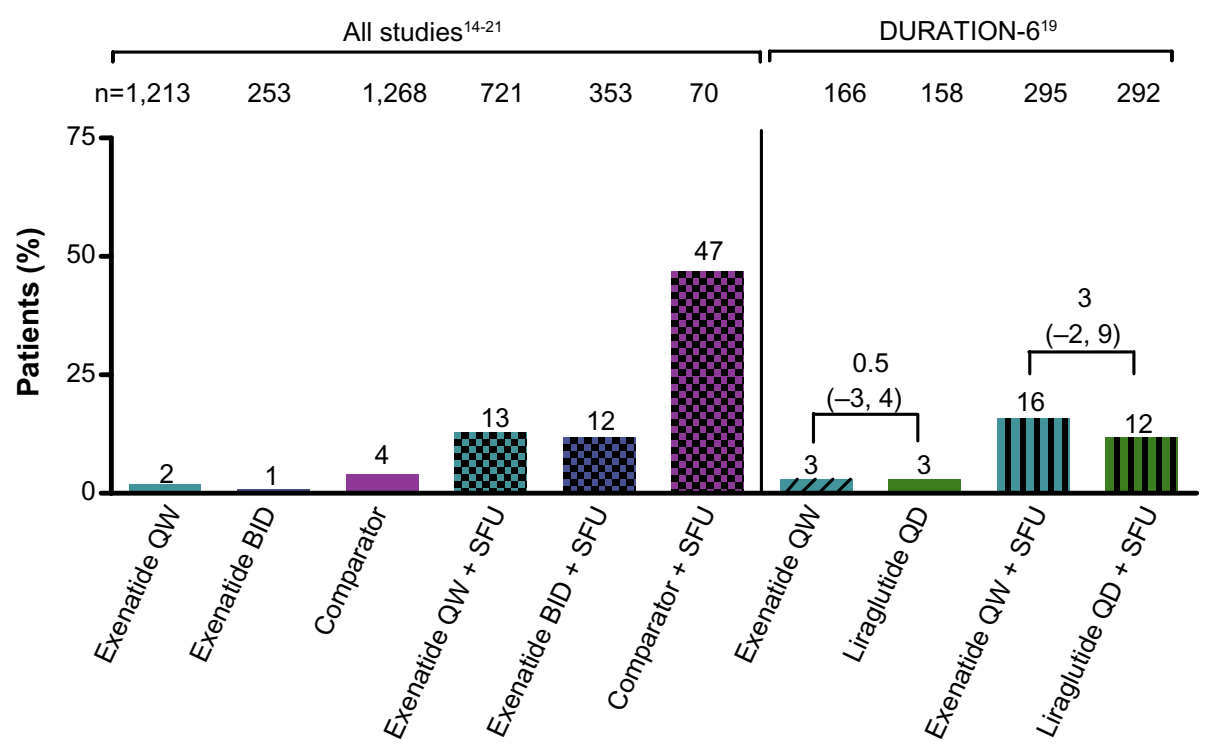

Figure 4 Incidence of hypoglycemia by treatment and use of SFU. Percentage of patients who experienced minor hypoglycemia. $95 \%$ confidence interval of the difference (exenatide QW incidence [\%] minus liraglutide QD incidence [\%] in DURATION-6).

Abbreviations: BID, twice daily; QD, once daily; QW, once weekly; SFU, sulfonylurea; DURATION, Diabetes Therapy Utilization: Researching Changes in AIC, Weight, and Other Factors Through Intervention With Exenatide Once Weekly.

liraglutide QD produced similar results with regard to the prevalence of GI-related AEs and the transient occurrence of nausea. ${ }^{4,26}$

Injection site-related AEs were numerically more commonly reported with exenatide QW therapy than with other GLP-1RAs or non-GLP-1RA comparators and infrequently required discontinuation. Occurrence of small asymptomatic injection site nodules was an expected event given the inherent characteristics of the microsphere delivery system. ${ }^{13}$ Small asymptomatic injection site nodules are commonly associated with the injection of drugs in the microsphere-based delivery method. However, these nodules are transient and resolve without medical intervention, regardless of antibody status.

A previous report published the incidence of antibody formation and the association of antibodies with injection site reactions in DUR-1, DUR-2, and DUR-3. ${ }^{32}$ A positive antibody titer was seen in $57 \%$ of patients at $26-30$ weeks $(45 \%$ low titer $[\leq 125], 12 \%$ higher titer $[\geq 625]$ ). The incidence of all potentially immune-related AEs in antibody-negative and antibody-positive patients was $12 \%$ and $22 \%$, respectively, in the exenatide QW groups and $10 \%$ in the pooled

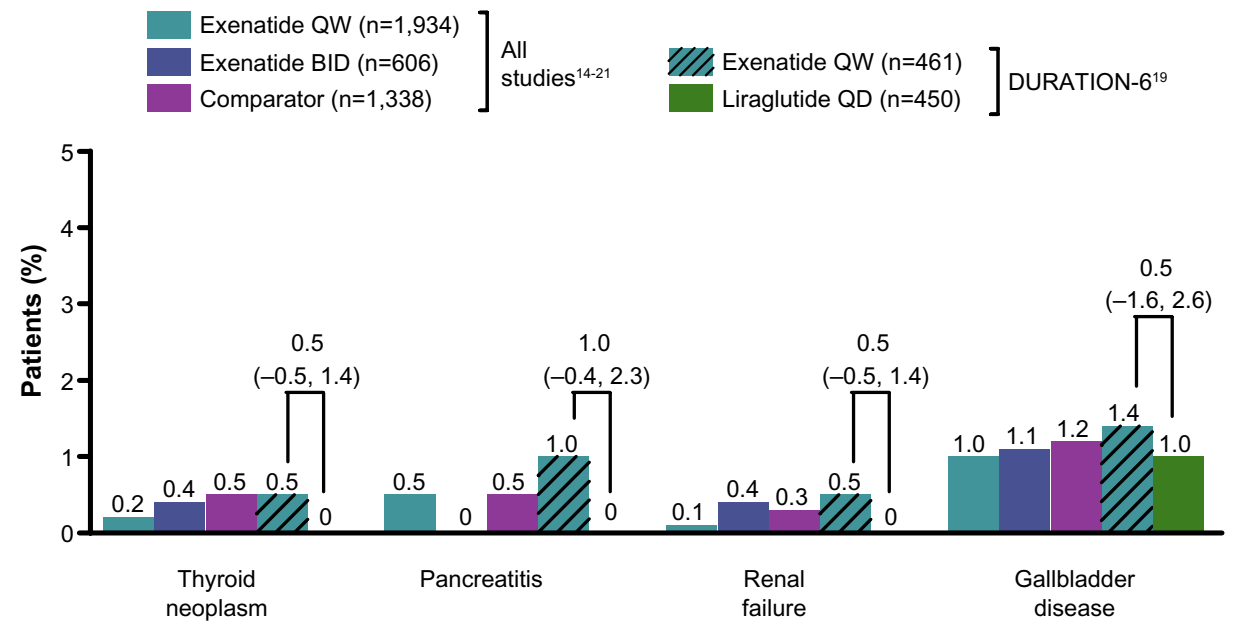

Figure 5 Adverse events of interest. Exposure-adjusted incidence per 100 patient-years and difference in thyroid neoplasm, pancreatitis, renal failure, and gallbladder disease. Pancreatitis includes acute pancreatitis and chronic pancreatitis. Thyroid neoplasm includes benign neoplasm of the thyroid gland and malignant thyroid neoplasm. $95 \%$ confidence interval for the difference (exenatide QW incidence [\%] minus liraglutide QD incidence [\%] in DURATION-6).

Abbreviations: BID, twice daily; QD, once daily; QW, once weekly; DURATION, Diabetes Therapy Utilization: Researching Changes in AIC, Weight, and Other Factors Through Intervention With Exenatide Once Weekly. 
Table 6 Treatment-emergent serious adverse events by body system ( $>1 \%$ in any group) for 24-week to 30-week randomized comparator-controlled trials ${ }^{14-21}$

\begin{tabular}{|c|c|c|c|c|c|}
\hline \multirow[t]{2}{*}{ System } & \multicolumn{5}{|c|}{ Incidence, n (\%) (preferred terms) } \\
\hline & $\begin{array}{l}\text { Pooled exenatide } \\
\text { QW }(n=1,934)\end{array}$ & $\begin{array}{l}\text { Pooled exenatide } \\
\text { BID }(n=606)\end{array}$ & $\begin{array}{l}\text { Pooled non-GLP-IRA } \\
\text { comparators }(n=I, 338)\end{array}$ & $\begin{array}{l}\text { Exenatide } \\
\text { QW (n=46I) }\end{array}$ & $\begin{array}{l}\text { Liraglutide } \\
\text { QD }(n=450)\end{array}$ \\
\hline Cardiac disorders & $8(0.4 \%)$ & $4(0.7 \%)$ & $9(0.7 \%)$ & I (0.2\%) & $2(0.4 \%)$ \\
\hline Gastrointestinal disorders & $9(0.5 \%)$ & $2(0.3 \%)$ & $8(0.6 \%)$ & $3(0.7 \%)$ & $2(0.4 \%)$ \\
\hline $\begin{array}{l}\text { General disorders and administration } \\
\text { site conditions }\end{array}$ & $\mathrm{I}(<0.1 \%)$ & $2(0.3 \%)$ & $7(0.5 \%)$ & $0(0.0 \%)$ & I (0.2\%) \\
\hline Hepatobiliary disorders & $5(0.3 \%)$ & $2(0.3 \%)$ & $6(0.4 \%)$ & $2(0.4 \%)$ & $0(0.0 \%)$ \\
\hline Infections and infestations & $12(0.6 \%)$ & $2(0.3 \%)$ & $12(0.9 \%)$ & $2(0.4 \%)$ & $0(0.0 \%)$ \\
\hline $\begin{array}{l}\text { Neoplasms benign, malignant, and } \\
\text { unspecified (including cysts and polyps) }\end{array}$ & $5(0.3 \%)$ & $0(0.0 \%)$ & $4(0.3 \%)$ & $2(0.4 \%)$ & $0(0.0 \%)$ \\
\hline Nervous system disorders & $6(0.3 \%)$ & I (0.2\%) & $6(0.4 \%)$ & $0(0.0 \%)$ & I (0.2\%) \\
\hline
\end{tabular}

Abbreviations: BID, twice daily; GLP-IRA, glucagon-like peptide-I receptor agonist; QD, once daily; QW, once weekly.

comparator group. Injection site erythema and pruritus occurred at a greater rate in antibody-positive patients than in antibody-negative patients with exenatide QW and in patients in the pooled comparator group (positive, $5 \%$ and $8 \%$, respectively; negative, $0.5 \%$ and $3 \%$; comparator, $1 \%$ and $2 \%) .{ }^{32}$ The incidence of all other injection site reactions was similar between groups.

Management of hypoglycemia is a concern with all glucose-lowering treatments. ${ }^{33,34}$ Major hypoglycemia was rare in all treatment groups, with four cases reported (one each in the pooled exenatide QW and BID groups and two in the pooled comparator group [insulin glargine]). Overall, the incidence of minor hypoglycemia was low across all groups without concomitant sulfonylurea therapy but occurred more frequently across all groups with concomitant sulfonylurea use. All events of hypoglycemia were transient.

The potential for increased risk of pancreatitis has been the subject of discussion for the GLP-1RA class. Further, concerns about pancreatitis with antihyperglycemic agents have been emphasized by postmarketing safety surveillance and case reports. ${ }^{35-37}$ In 2007 and 2008, the US FDA issued a safety alert and update based on postmarketing surveillance that reported a total of 36 cases of acute pancreatitis in patients treated with exenatide. ${ }^{38}$ However, pharmacovigilance data from the FDA Adverse Event Reporting System should be interpreted with caution given the varied reporting of supporting information and the absence of adjudication. ${ }^{39}$ Patients with type 2 diabetes appear to be at an increased risk for pancreatitis, as shown by large studies of health care databases. ${ }^{40-42}$ To date, the majority of large postmarketing studies ${ }^{42-45}$ have found no evidence to support an association between exenatide and increased risk of pancreatitis. In one study, the incidence of acute pancreatitis $(0.13 \%)$ was comparable for initiators of exenatide relative to the comparator group $(0.12 \%$; relative risk $1.0 ; 95 \%$ confidence interval $0.6-1.7) ;{ }^{43}$ in a second study, no definite association could be found between use of exenatide and increased incidence of pancreatitis; ${ }^{42}$ and in a third study, use of GLP-1-based therapies was associated with an increased risk of acute pancreatitis relative to nonusers. ${ }^{45}$ Other glucose-lowering therapies for the treatment of type 2 diabetes have also been associated with the development of pancreatitis, ${ }^{46}$ although such occurrences have been rare. ${ }^{47-49}$ Recently, the FDA and European Medicines Agency conducted parallel assessments of the pancreatic safety of incretin-based drugs (based on nonclinical toxicology studies, clinical trial data, and epidemiological data) and did not find data consistent with previous reports of a causal association between incretinbased drugs and pancreatitis or pancreatic cancer. ${ }^{50}$ In the present analysis, events were rare in all groups (Table 6). There was no apparent trend for exenatide QW in extending the clinical course of pancreatitis, and events resolved with and without continued use of exenatide QW. Ongoing observational studies continue to assess the pancreatic safety of incretin-based therapies.

Based on preclinical studies, exenatide is not directly nephrotoxic as it is eliminated by glomerular filtration and subsequent proteolytic degradation in the renal tubules, resulting in no active metabolites. ${ }^{12,51}$ Chronic kidney disease is common in patients with type 2 diabetes, and postmarketing case reports of an association between use of exenatide and worsened kidney function raised concerns over a causal relationship. Because exenatide is primarily cleared via renal mechanisms,${ }^{51}$ the exenatide QW label contains a warning that it should not be used in patients with severe renal impairment (creatinine clearance $<30 \mathrm{~mL} / \mathrm{min}$ ) or end-stage renal disease, should be used with caution in patients with renal transplantation, and caution should be applied when initiating 
or escalating doses of exenatide in patients with moderate renal impairment (creatinine clearance 30-50 mL/min). ${ }^{12}$ Additionally, because exenatide may induce nausea and vomiting with transient hypovolemia, treatment may worsen renal function in these instances because of decreased renal perfusion. In these circumstances, rehydration usually restores renal function to baseline values. Events of exenatide-associated renal impairment or renal failure are reported infrequently in the published studies. ${ }^{52,53}$ In the current analysis, renal failure was rarely reported. These data are consistent with six clinical trials ${ }^{54}$ that found no association between exenatide BID or placebo in renal AEs and similar changes in kidney function between patients in both groups.

Thyroid cancers are of some interest with the GLP-1RAs, as sustained GLP-1 agonism has been associated with an increased incidence of C-cell adenomas and carcinomas in rodents; ${ }^{55}$ however, the clinical relevance of these animal data is unknown. In particular, there appears to be a speciesspecific difference in thyroid C-cell response to GLP-1RAs that causes $\mathrm{C}$-cell secretion of calcitonin and hyperplasia in rodents. ${ }^{55}$ In the present analysis, all occurrences of thyroid neoplasm were benign and very rare, and no C-cell cancers were reported in these clinical trials. Thyroid neoplasm has rarely been reported with liraglutide QD, and studies monitoring calcitonin have indicated similar levels with liraglutide QD and comparators. ${ }^{56-58}$ A US insurance claims database showed no significant increased risk of inpatient pancreatic or thyroid cancer between exenatide BID and metformin or glyburide. ${ }^{43}$ Ongoing postmarketing surveillance of exenatide shows no indication of a safety signal. ${ }^{59}$ Overall, the incidence of thyroid neoplasm-related events across treatments is consistent with the background rate of thyroid cancers in the general population.

FDA guidance requires new treatments for type 2 diabetes to demonstrate that treatment will not result in an unacceptable cardiovascular risk. ${ }^{60}$ While cardiovascular safety data for exenatide QW and comparators have been limited by a short follow-up duration, in an evaluation of the change in heart rate over time a small increase in heart rate $(+1.3 \mathrm{bpm})$ was observed upon starting exenatide QW therapy; after 26 weeks of treatment, the mean change was $+2.6 \mathrm{bpm}$. Heart rate returned to near baseline levels 10-12 weeks following discontinuation of exenatide QW. ${ }^{61}$ These data are consistent with demonstrated heart rate changes as observed in previous reports, ${ }^{62}$ and no increase in cardiovascular event rates for exenatide QW, exenatide
BID, or comparators as observed in previous studies of GLP-1RAs. ${ }^{63}$ Moreover, diabetes increases the risk of cardiovascular disease in men and women. ${ }^{64}$ Our analysis of primary and expanded MACE in this combined analysis show that the rate of these events was low $(<1.0 \%)$ for patients treated with exenatide QW. Studies evaluating cardiovascular outcomes with exenatide, liraglutide, dulaglutide, and lixisenatide are ongoing. The EXSCEL trial (ClinicalTrials.gov identifier NCT01144338) is evaluating the impact of including exenatide QW as part of usual care versus usual care without exenatide on major cardiovascular outcomes as measured by the primary cardiovascular combined endpoint of cardiovascular-related death, nonfatal MI, or nonfatal stroke in approximately 14,000 patients with type 2 diabetes.

Antibodies to GLP-1RAs may develop in some patients, as has been observed with other peptide therapeutics. ${ }^{56,65}$ Furthermore, formation of antibodies to therapeutic peptides is common, even when the peptide is identical to the endogenous human form. In a study by Fineman et al, ${ }^{32} 37 \%$ of exenatide-treated patients developed antibodies to exenatide after 30 weeks of treatment; this rate fell to $17 \%$ after 3 years. In a study of liraglutide QD, antibodies developed in approximately $8 \%$ of patients who received liraglutide QD for up to 26 weeks. ${ }^{66}$ Analysis of cross-reactivity in a subset of antibody-positive patients in the Buse et al study showed that treatment-emergent antibodies to exenatide did not cross-react with human GLP-1 or glucagon. Similarly, in the LEAD-6 trial of liraglutide QD, 4.4\% ( $n=5 / 113)$ of antibody-positive samples cross-reacted with GLP-1; however, it was unknown whether the cross-reactivity was pre-existing or treatment-emergent. ${ }^{67} \mathrm{~A}$ low antibody titer does not appear to be predictive of safety or efficacy issues as there was no difference between groups in potentially immune-related AEs overall, and only a slight increase in the occurrence of some injection site-related AEs in patients with a positive antibody titer to exenatide. ${ }^{32}$ There were no reports of systemic hypersensitivity or immune-related respiratory reactions such as anaphylaxis with exenatide QW treatment.

In open-label extension periods up to 3 years, exenatide QW maintained improvements in glycemic control and weight loss when compared with exenatide BID or insulin glargine. ${ }^{68,69}$ The AEs reported in these longer trials are similar to those observed in this integrated safety analysis. Moreover, the AEs were mostly mild in intensity and decreased over time. 


\section{Strengths and limitations}

There are several strengths of this analysis: a large number of patients were included in the pooled data set; the trials were randomized and controlled with centralized monitoring and laboratory analyses; and results were derived from individual patient data. Limitations of this analysis are that AEs were not independently adjudicated and the post hoc design of this analysis was not adequately powered to detect very rare AEs (incidence $<0.01 \%$ ), nor were the duration of the trials long enough to observe rare AEs with a long course of development (eg, cancers). Patients with clinically significant comorbidities were excluded from participation in the studies that comprised the pooled analysis; thus, these data should not be generalized to such patients. Although the trials were similarly designed with respect to randomization and blinding, there were concerns about drawing comparisons between pooled treatment groups because of the heterogeneity in the patient population and other aspects of study design.

\section{Conclusion}

In this integrated analysis of more than 4,328 patients studied for up to 6 months and representing over 2,100 patient-years of exposure, exenatide QW was generally well tolerated and had an acceptable safety profile in patients with type 2 diabetes. Consistent with previous studies, the primary apparent difference in the GLP-1RAs compared with the nonGLP-1RA comparators was in GI tolerability. This analysis further showed no apparent differences in the overall safety and tolerability profiles of the GLP-1RAs across treatments, despite differences in the drug profiles (ie, short-acting versus intermediate-acting versus long-acting). Exenatide QW was associated with improved GI tolerability compared with exenatide BID or liraglutide QD; however, injection site reactions were more frequent with exenatide $\mathrm{QW}$.

\section{Acknowledgments}

These studies were sponsored by Amylin Pharmaceuticals, LLC, and Eli Lilly and Company. We thank the patients, investigators, and their staff for participating in the included studies. We also thank Jenny Han (Amylin, Bristol-Myers Squibb) and Haiying Dong (Amylin) for their contributions to the data analysis, Rita Petroro (Bristol-Myers Squibb) for her contribution to the safety analysis, and Carmelle Remillard (Amylin), Julie Ellison (Amylin, Bristol-Myers Squibb), and Mary Beth DeYoung (Amylin, Bristol-Myers Squibb, AstraZeneca) for reviewing and editing the manuscript. Lisa M Klumpp Callan and Sushma Soni of inScience
Communications, Springer Healthcare, provided medical writing support funded by AstraZeneca.

\section{Author contributions}

LM, JM, and OK contributed to the individual study designs of the trials included in this analysis. LM and JM contributed to the conception and design of the present analysis, and all authors contributed to interpretation of the data and critical revision of the manuscript. MZ performed the data analysis. All authors approved the final version to be published and agree to be accountable for the work.

\section{Disclosure}

LM, JM, and MZ were employees of Bristol-Myers Squibb and OK was an employee of Amylin Pharmaceuticals, LLC, at the time these studies were conducted. KG is an employee and shareholder of Bristol-Myers Squibb Company. The authors report no other conflicts of interest in this work.

\section{References}

1. American Diabetes Association. Standards of medical care in diabetes - 2015. Diabetes Care. 2015;38 Suppl 1:S1-S94.

2. Garber AJ, Abrahamson MJ, Barzilay JI, et al. American Association of Clinical Endocrinologists' comprehensive diabetes management algorithm 2013 consensus statement. Endocr Pract. 2013;19 Suppl 2: 1-48.

3. Inzucchi SE, Bergenstal RM, Buse JB, et al. Management of hyperglycemia in type 2 diabetes, 2015: a patient-centered approach: update to a position statement of the American Diabetes Association and the European Association for the Study of Diabetes. Diabetes Care. 2015; 38(1):140-149.

4. Blonde L, Russell-Jones D. The safety and efficacy of liraglutide with or without oral antidiabetic drug therapy in type 2 diabetes: an overview of the LEAD 1-5 studies. Diabetes Obes Metab. 2009;11 Suppl 3:26-34.

5. Grimm M, Han J, Weaver C, et al. Efficacy, safety, and tolerability of exenatide once weekly in patients with type 2 diabetes mellitus: an integrated analysis of the DURATION trials. Postgrad Med. 2013;125(3): 47-57.

6. Trujillo JM, Nuffer W. GLP-1 receptor agonists for type 2 diabetes mellitus: recent developments and emerging agents. Pharmacotherapy. 2014;34(11):1174-1186.

7. Fineman M, Flanagan S, Taylor K, et al. Pharmacokinetics and pharmacodynamics of exenatide extended-release after single and multiple dosing. Clin Pharmacokinet. 2011;50(1):65-74.

8. Nauck MA, Holst JJ, Willms B, Schmiegel W. Glucagon-like peptide 1 (GLP-1) as a new therapeutic approach for type 2-diabetes. Exp Clin Endocrinol Diabetes. 1997;105(4):187-195.

9. MacConell L, Brown C, Gurney K, Han J. Safety and tolerability of exenatide twice daily in patients with type 2 diabetes: integrated analysis of 5594 patients from 19 placebo-controlled and comparator-controlled clinical trials. Diabetes Metab Syndr Obes. 2012;5:29-41.

10. Montanya E, Sesti G. A review of efficacy and safety data regarding the use of liraglutide, a once-daily human glucagon-like peptide 1 analogue, in the treatment of type 2 diabetes mellitus. Clin Ther. 2009;31(11): 2472-2488.

11. Bode BW, Testa MA, Magwire M, et al. Patient-reported outcomes following treatment with the human GLP-1 analogue liraglutide or glimepiride in monotherapy: results from a randomized controlled trial in patients with type 2 diabetes. Diabetes Obes Metab. 2010;12(7): 604-612. 
12. Bydureon (exenatide extended-release for injectable suspension): US prescribing information (revised May 2014). Wilmington, DE, USA: AstraZeneca Pharmaceuticals LP; 2014.

13. DeYoung MB, MacConell L, Sarin V, Trautmann M, Herbert P. Encapsulation of exenatide in poly-(D,L-lactide-co-glycolide) microspheres produced an investigational long-acting once-weekly formulation for type 2 diabetes. Diabetes Technol Ther. 2011;13(11):1145-1154.

14. Drucker DJ, Buse JB, Taylor K, et al. Exenatide once weekly versus twice daily for the treatment of type 2 diabetes: a randomised, openlabel, non-inferiority study. Lancet. 2008;372(9645):1240-1250.

15. Bergenstal RM, Wysham C, MacConell L, et al. Efficacy and safety of exenatide once weekly versus sitagliptin or pioglitazone as an adjunct to metformin for treatment of type 2 diabetes (DURATION-2): a randomised trial. Lancet. 2010;376(9739):431-439.

16. Diamant M, Van Gaal L, Stranks S, et al. Once weekly exenatide compared with insulin glargine titrated to target in patients with type 2 diabetes (DURATION-3): an open-label randomised trial. Lancet. 2010;375(9733):2234-2243.

17. Russell-Jones D, Cuddihy RM, Hanefeld M, et al. Efficacy and safety of exenatide once weekly versus metformin, pioglitazone, and sitagliptin used as monotherapy in drug-naive patients with type 2 diabetes (DURATION-4): a 26-week double-blind study. Diabetes Care. 2012; 35(2):252-258.

18. Blevins T, Pullman J, Malloy J, et al. DURATION-5: exenatide once weekly resulted in greater improvements in glycemic control compared with exenatide twice daily in patients with type 2 diabetes. J Clin Endocrinol Metab. 2011;96(5):1301-1310.

19. Buse JB, Nauck M, Forst T, et al. Exenatide once weekly versus liraglutide once daily in patients with type 2 diabetes (DURATION-6): a randomised, open-label study. Lancet. 2013;381(9861):117-124.

20. Inagaki N, Atsumi Y, Oura T, Saito H, Imaoka T. Efficacy and safety profile of exenatide once weekly compared with insulin once daily in Japanese patients with type 2 diabetes treated with oral antidiabetes $\operatorname{drug}(\mathrm{s})$ : results from a 26-week, randomized, open-label, parallelgroup, multicenter, noninferiority study. Clin Ther. 2012;34(9): 1892-1908.

21. Ji L, Onishi Y, Ahn CW, et al. Efficacy and safety of exenatide onceweekly vs exenatide twice-daily in Asian patients with type 2 diabetes mellitus. J Diabetes Investig. 2013;4(1):53-61.

22. World Medical Association. Declaration of Helsinki: Ethical principles for medical research involving human subjects. 2008. Available from: http://www.wma.net/en/30publications/10policies/b3/index.html. Accessed September 25, 2014.

23. Bytzer P, Talley NJ, Leemon M, Young LJ, Jones MP, Horowitz M. Prevalence of gastrointestinal symptoms associated with diabetes mellitus: a population-based survey of 15,000 adults. Arch Intern Med. 2001;161(16):1989-1996.

24. Icks A, Haastert B, Rathmann W, Wareham N. Prevalence of gastrointestinal symptoms in patients with type 2 diabetes: a population-based study. Arch Intern Med. 2002;162(9):1067-1069.

25. Kim JH, Park HS, Ko SY, et al. Diabetic factors associated with gastrointestinal symptoms in patients with type 2 diabetes. World $J$ Gastroenterol. 2010;16(14):1782-1787.

26. Horowitz M, Vilsboll T, Zdravkovic M, Hammer M, Madsbad S. Patient-reported rating of gastrointestinal adverse effects during treatment of type 2 diabetes with the once-daily human GLP-1 analogue, liraglutide. Diabetes Obes Metab. 2008;10(7):593-596.

27. Astrup A, Rossner S, Van Gaal L, et al. Effects of liraglutide in the treatment of obesity: a randomised, double-blind, placebo-controlled study. Lancet. 2009;374(9701):1606-1616.

28. Davidson JA, Nikkel C, Grimm M. Exenatide once weekly: opportunities in the primary care setting. Postgrad Med. 2013;125(3):68-78.

29. Klonoff DC, Buse JB, Nielsen LL, et al. Exenatide effects on diabetes, obesity, cardiovascular risk factors and hepatic biomarkers in patients with type 2 diabetes treated for at least 3 years. Curr Med Res Opin. 2008;24(1):275-286.
30. Buse JB, Klonoff DC, Nielsen LL, et al. Metabolic effects of two years of exenatide treatment on diabetes, obesity, and hepatic biomarkers in patients with type 2 diabetes: an interim analysis of data from the openlabel, uncontrolled extension of three double-blind, placebo-controlled trials. Clin Ther. 2007;29(1):139-153.

31. Bunck MC, Diamant M, Corner A, et al. One-year treatment with exenatide improves beta-cell function, compared with insulin glargine, in metformin-treated type 2 diabetic patients: a randomized, controlled trial. Diabetes Care. 2009;32(5):762-768.

32. Fineman MS, Mace KF, Diamant M, et al. Clinical relevance of antiexenatide antibodies: safety, efficacy and cross-reactivity with long-term treatment. Diabetes Obes Metab. 2012;14(6):546-554.

33. McCall AL, Cox DJ, Brodows R, Crean J, Johns D, Kovatchev B. Reduced daily risk of glycemic variability: comparison of exenatide with insulin glargine. Diabetes Technol Ther. 2009;11(6):339-344.

34. Wright AD, Cull CA, Macleod KM, Holman RR. Hypoglycemia in type 2 diabetic patients randomized to and maintained on monotherapy with diet, sulfonylurea, metformin, or insulin for 6 years from diagnosis: UKPDS73. J Diabetes Complications. 2006;20(6):395-401.

35. Denker PS, Dimarco PE. Exenatide (exendin-4)-induced pancreatitis: a case report. Diabetes Care. 2006;29(2):471.

36. Tripathy NR, Basha S, Jain R, Shetty S, Ramachandran A. Exenatide and acute pancreatitis. J Assoc Physicians India. 2008;56:987-988.

37. Ayoub WA, Kumar AA, Naguib HS, Taylor HC. Exenatide-induced acute pancreatitis. Endocr Pract. 2010;16(1):80-83.

38. US Food and Drug Administration. Information for Healthcare Professionals: exenatide (marketed as Byetta). 2008. Available from: http://www.fda.gov/Drugs/DrugSafety/PostmarketDrugSafetyInformation forPatientsandProviders/ucm124713.htm. Accessed September 25, 2014.

39. Raschi E, Piccinni C, Poluzzi E, Marchesini G, De Ponti F. The association of pancreatitis with antidiabetic drug use: gaining insight through the FDA pharmacovigilance database. Acta Diabetol. 2013;50(4): 569-577.

40. Noel RA, Braun DK, Patterson RE, Bloomgren GL. Increased risk of acute pancreatitis and biliary disease observed in patients with type 2 diabetes: a retrospective cohort study. Diabetes Care. 2009;32(5): 834-838.

41. Girman CJ, Kou TD, Cai B, et al. Patients with type 2 diabetes mellitus have higher risk for acute pancreatitis compared with those without diabetes. Diabetes Obes Metab. 2010;12(9):766-771.

42. Garg R, Chen W, Pendergrass M. Acute pancreatitis in type 2 diabetes treated with exenatide or sitagliptin: a retrospective observational pharmacy claims analysis. Diabetes Care. 2010;33(11): 2349-2354.

43. Dore DD, Seeger JD, Arnold Chan K. Use of a claims-based active drug safety surveillance system to assess the risk of acute pancreatitis with exenatide or sitagliptin compared to metformin or glyburide. Curr Med Res Opin. 2009;25(4):1019-1027.

44. Romley JA, Goldman DP, Solomon M, McFadden D, Peters AL. Exenatide therapy and the risk of pancreatitis and pancreatic cancer in a privately insured population. Diabetes Technol Ther. 2012;14(10): 904-911.

45. Singh S, Chang HY, Richards TM, Weiner JP, Clark JM, Segal JB. Glucagonlike peptide 1-based therapies and risk of hospitalization for acute pancreatitis in type 2 diabetes mellitus: a population-based matched case-control study. JAMA Intern Med. 2013;173(7):534-539.

46. US Food and Drug Administration. Information for Healthcare Professionals: acute pancreatitis and sitagliptin (marketed as Januvia and Janumet). 2009. Available from: http://www.fda.gov/Drugs/ DrugSafety/PostmarketDrugSafetyInformationforPatientsandProvide rs/DrugSafetyInformationforHeathcareProfessionals/ucm183764.htm. Accessed September 25, 2014.

47. Blomgren KB, Sundstrom A, Steineck G, Wiholm BE. Obesity and treatment of diabetes with glyburide may both be risk factors for acute pancreatitis. Diabetes Care. 2002;25(2):298-302. 
48. Fimognari FL, Corsonello A, Pastorell R, Antonelli-Incalzi R. Metformin-induced pancreatitis: a possible adverse drug effect during acute renal failure. Diabetes Care. 2006;29(5):1183.

49. Gonzalez-Perez A, Schlienger RG, Rodriguez LA. Acute pancreatitis in association with type 2 diabetes and antidiabetic drugs: a populationbased cohort study. Diabetes Care. 2010;33(12):2580-2585.

50. Egan AG, Blind E, Dunder K, et al. Pancreatic safety of incretin-based drugs - FDA and EMA assessment. N Engl J Med. 2014;370(9): 794-797.

51. Copley K, McCowen K, Hiles R, Nielsen LL, Young A, Parkes DG. Investigation of exenatide elimination and its in vivo and in vitro degradation. Curr Drug Metab. 2006;7(4):367-374.

52. Ferrer-Garcia JC, Martinez-Chanza N, Tolosa-Torrens M, Sanchez-Juan C. Exenatide and renal failure. Diabet Med. 2010;27(6):728-729.

53. Weise WJ, Sivanandy MS, Block CA, Comi RJ. Exenatide-associated ischemic renal failure. Diabetes Care. 2009;32(2):e22-e23.

54. Tuttle KR, Heilmann C, Hoogwerf BJ, Brown C, Anderson PW. Effects of exenatide on kidney function, adverse events, and clinical end points of kidney disease in type 2 diabetes. Am J Kidney Dis. 2013;62(2): 396-398.

55. Bjerre Knudsen L, Madsen LW, Andersen S, et al. Glucagon-like peptide-1 receptor agonists activate rodent thyroid C-cells causing calcitonin release and C-cell proliferation. Endocrinology. 2010;151(4): 1473-1486.

56. Buse JB, Rosenstock J, Sesti G, et al. Liraglutide once a day versus exenatide twice a day for type 2 diabetes: a 26-week randomised, parallel-group, multinational, open-label trial (LEAD-6). Lancet. 2009; 374(9683):39-47.

57. Garber A, Henry R, Ratner R, et al. Liraglutide versus glimepiride monotherapy for type 2 diabetes (LEAD-3 Mono): a randomised, 52-week, phase III, double-blind, parallel-treatment trial. Lancet. 2009; 373(9662):473-481.

58. Hegedus L, Moses AC, Zdravkovic M, Le Thi T, Daniels GH. GLP-1 and calcitonin concentration in humans: lack of evidence of calcitonin release from sequential screening in over 5000 subjects with type 2 diabetes or nondiabetic obese subjects treated with the human GLP-1 analog, liraglutide. J Clin Endocrinol Metab. 2011;96(3):853-860.

59. Dore DD, Seeger JD, Chan KA. Incidence of health insurance claims for thyroid neoplasm and pancreatic malignancy in association with exenatide: signal refinement using active safety surveillance. Ther $A d v$ Drug Saf. 2012;3(4):157-164.
60. US Department of Health and Human Services Center for Drug Evaluation and Research. Guidance for industry: diabetes mellitus - evaluating cardiovascular risk in new antidiabetic therapies to treat type 2 diabetes. 2008. Available from: http://www.fda.gov/downloads/Drugs/ GuidanceComplianceRegulatoryInformation/Guidances/ucm071627. pdf. Accessed September 25, 2014.

61. Chilton RJ, MacConell LA, Han JC, Marso SP. Characterization of heart rate increases with glucagon-like peptide-1 agonist therapy. Circulation. 2013;128:A16290.

62. Robinson LE, Holt TA, Rees K, Randeva HS, O'Hare JP. Effects of exenatide and liraglutide on heart rate, blood pressure and body weight: systematic review and meta-analysis. BMJ Open. 2013;3(1).

63. Monami M, Cremasco F, Lamanna C, et al. Glucagon-like peptide-1 receptor agonists and cardiovascular events: a meta-analysis of randomized clinical trials. Exp Diabetes Res. 2011;2011:215764.

64. Grundy SM, Pasternak R, Greenland P, Smith S Jr, Fuster V. Assessment of cardiovascular risk by use of multiple-risk-factor assessment equations: a statement for healthcare professionals from the American Heart Association and the American College of Cardiology. Circulation . 1999;100(13):1481-1492.

65. Russell-Jones D, Vaag A, Schmitz O, et al. Liraglutide vs insulin glargine and placebo in combination with metformin and sulfonylurea therapy in type 2 diabetes mellitus (LEAD-5 met+SU): a randomised controlled trial. Diabetologia. 2009;52(10):2046-2055.

66. Buse JB, Garber A, Rosenstock J, et al. Liraglutide treatment is associated with a low frequency and magnitude of antibody formation with no apparent impact on glycemic response or increased frequency of adverse events: results from the Liraglutide Effect and Action in Diabetes (LEAD) trials. J Clin Endocrinol Metab. 2011;96(6):1695-1702.

67. Buse J, Montanya E, Sesti G, et al. Frequency and magnitude of antibody formation are lower with liraglutide than exenatide: LEAD-6 results. Diabetes Care. 2010;59 Suppl 1:A184.

68. MacConell L, Pencek R, Li Y, Maggs D, Porter L. Exenatide once weekly: sustained improvement in glycemic control and cardiometabolic measures through 3 years. Diabetes Metab Syndr Obes. 2013;6: $31-41$.

69. Diamant M, Van Gaal L, Guerci B, et al. Exenatide once weekly versus insulin glargine for type 2 diabetes (DURATION-3): 3-year results of an open-label randomised trial. Lancet Diabetes Endocrinol. 2014;2(6): 464-473.

\section{Publish your work in this journal}

Diabetes, Metabolic Syndrome and Obesity: Targets and Therapy is an international, peer-reviewed open-access journal committed to the rapid publication of the latest laboratory and clinical findings in the fields of diabetes, metabolic syndrome and obesity research Original research, review, case reports, hypothesis formation, expert opinion and commentaries are all considered for publication. The manuscript management system is completely online and includes a very quick and fair peer-review system, which is all easy to use. Visit http://www.dovepress.com/testimonials.php to read real quotes from published authors. 